\title{
Modelo LOGIT com fatores sociais para gravidade de acidentes com motocicletas
}

\author{
Logit model with social factors for motorcycle accident gravity
}

\author{
Jessica Helena de Lima ${ }^{1}$, Anderson Moreira Aristides dos Santos ${ }^{2}$, Maria Leonor Alves Maia ${ }^{3}$ \\ ${ }^{1}$ Universidade Federal de Alagoas, Alagoas - Brasil, delima.jh@gmail.com \\ 2Universidade Federal de Alagoas, Alagoas - Brasil, anderson.santos@feac.ufal.br \\ 3Universidade Federal de Pernambuco, Pernambuco - Brasil, nonamaia@gmail.com
}

\section{Recebido: \\ 9 de abril de 2020 \\ 18 de agosto de 2020 \\ Publicado: \\ 23 de julho de 2021 \\ Editor de área: \\ Palavras-chave: \\ Motocicleta. \\ Transporte rural. \\ Acidente. \\ Inclusão social. \\ Logit. \\ Keywords: \\ Motorcycle. \\ Rural transport. \\ Accidents. \\ Social inclusion. \\ Logit.}

Aceito para publicação:

Helena Beatriz Bettella Cybis

DOI:10.14295/transportes.v29i1.2349

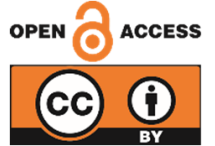

\begin{abstract}
RESUMO
Objetiva-se avaliar se os atributos socioeconômicos das vítimas, como escolaridade, renda e gênero são determinantes em aumentar a probabilidade de envolvimento em acidentes fatais. Um modelo logit stepwise foi utilizado para determinar os fatores que influenciam na gravidade de acidentes de trânsito. Foram utilizados 689 questionários com dados socioeconômicos e, também, dados relativos ao acidente, de solicitantes do seguro DPVAT no estado de Alagoas, entre 2013 e 2019, para estimar o modelo. Os resultados mostram que são significativas as variáveis sexo, escolaridade, tipo de motocicleta, uso de capacete, ser habilitado e acidentes em finais de semana. Ou seja, além dos fatores relativos ao acidente, fatores inerentes ao grupo social do indivíduo influenciam na sua probabilidade de morte. Conhecer tais fatores é crucial para identificar os grupos sociais mais vulneráveis a mortalidade no trânsito e elaborar políticas públicas direcionadas a eles.
\end{abstract}

\section{ABSTRACT}

This paper aims to assess whether the victims' socioeconomic attributes, such as education, income and gender, are decisive in increasing the likelihood of being involved in a fatal crash. A stepwise logit model was used to determine factors that influence traffic accident's severity. 689 questionnaires with socioeconomic and crash related data, from DPVAT (Brazil's crash victims' universal insurance) applicants from the state of Alagoas, between 2013 and 2019 were used to estimate the model. Results show that the variables sex, education, motorcycle size, helmet, being licensed and accidents on weekends are significant. That is, in addition to crash related factors, factors inherent to the individual's social group influence his/her probability of death. Knowing such factors is crucial to identify the social groups that are most vulnerable to traffic mortality and thus develop focused public policies.

\section{INTRODUCÃO}

No mundo, o número de óbitos de acidentes de transporte terrestre está estabilizado em 1,25 milhão de pessoas por ano. 0 custo destes acidentes representa, em média, 3\% do PIB dos países, podendo chegar a 5\% em alguns casos. Atualmente, 90\% dos óbitos acontecem em países com rendimentos baixos ou médios que, em conjunto, possuem pouco mais da metade (54\%) da frota veicular motorizada do mundo (OMS, 2015).

No Brasil, entre 1998 e 2018, foram registrados 734.938 óbitos por acidentes de trânsito, o que, em intervalos de tempo, significa uma morte a cada 15 minutos. Este número ultrapassa o de homicídios por armas de fogo (726 mil) no mesmo período (MS, 2019). Analisando as 
tendências nacionais das estatísticas de acidentes entre os anos de 1996 e 2011, percebe-se: (i) quedas significativas na mortalidade de pedestres; (ii) pequeno aumento da letalidade de ocupantes de automóveis; e, (iii) grandes aumentos na mortalidade de motociclistas que, em 2014, representavam 38\% do total de vítimas fatais (Waiselfisz, 2013; MS, 2016). Em 2018, 64\% das indenizações por morte foram pagas a ocupantes de motocicleta (Seguradora Líder, 2019).

Sabendo que os motociclistas compõem um grupo de risco relevante, é importante identificar quais características socioeconômicas podem influenciar na probabilidade de óbito, de forma a direcionar políticas públicas mais efetivas para evitá-las. Em extensa revisão da literatura de acidentes com motocicletas, Vlahogianni et al. (2012) dividiram os fatores de risco presentes em 71 estudos no contexto da Europa, Ásia, Oceania e América do Norte entre quatro grupos: a) comportamentais; b) de infraestrutura; c) veiculares, e; d) climáticos. Dentre os fatores comportamentais podem ser citados: atitudes e padrões de condução; erros e descumprimento das leis de trânsito; falta de percepção das motos pelos motoristas dos carros; idade, gênero e experiência; educação e aprendizado; fadiga, álcool e outras deficiências; e, uso de equipamento de segurança. Nenhum dos estudos listados por Vlahogianni et al. (2012) considerou a renda e apenas um considerou escolaridade. Desde então, autores da área tem se debruçado sobre a importância dos fatores socias, tendo Factor et al. (2008), Goel et al. (2018) e Borhan et al. (2018), considerado, dentre outras variáveis, o nível de escolaridade; Sexton et al. (2004) e Crundall et al. (2013), considerado a experiência em conduzir; e Borhan et al. (2018) avaliado também os impactos da renda na probabilidade de envolvimento em acidentes graves.

No Brasil, autores como Coutinho et al. (2015) e Berger (2014) utilizaram modelos econométricos com algumas variáveis socioeconômicas para acidentes com motociclistas. Mas, não foram encontrados trabalhos nacionais que considerem a relação entre características socioeconômicas como sexo, renda, e escolaridade e a probabilidade de envolvimento de motociclistas em acidentes graves, avaliando criticamente a sua importância para a formulação de políticas públicas. Desta forma, este trabalho contribui para o avanço do estudo de acidentes de trânsito no Brasil, ao avaliar se características socioeconômicas impactam a gravidade dos acidentes permitindo entender se existem grupos que são mais susceptíveis, e portanto, mais vulneráveis ao envolvimento em acidentes graves e apontar a necessidade de campanhas de prevenção e mitigação específicas a estes grupos.

As variações de renda, escolaridade e educação no trânsito dificilmente são coletadas pelas bases de dados dos boletins de ocorrência da polícia ou de ambulâncias, amplamente utilizadas para estudos de acidentes com microdados. Além disso, tais bases, tendem a ser incompletas e enviesadas, pois, no momento do acidente, o foco é salvar vidas e não preencher formulários (Amoros et al. 2006; Vlahogianni et al. 2012). Este trabalho utiliza uma base de dados de 1220 questionários com informações sociais e relativas ao acidente de solicitantes do seguro DPVAT na sede do Detran no estado de Alagoas. Os formulários são preenchidos posteriormente ao acidente, permitindo a coleta mais detalhada dos dados, diferentemente de bases cujos formulários são preenchidos durante o socorro das vítimas. Foram considerados na análise os 689 questionários relativos a usuários de motocicleta, no período de 2013 a 2019, visando estimar um modelo multivariado de gravidade de acidentes de transporte considerando, além dos fatores sociais (ex. gênero, escolaridade, educação no trânsito, renda), fatores relacionados ao veículo e ao acidente (ex. uso de capacete, tipo de motocicleta).

As informações levantadas têm o potencial de auxiliar o poder público a focar suas políticas de prevenção e educação no trânsito no grupo identificado como de maior risco e, desta forma, 
obter uma maior efetividade no resguardo das vidas das prováveis vítimas. Desta forma, este estudo é essencial para fundamentar políticas públicas de prevenção de acidentes com motociclistas, usuários com o maior número de ocorrências graves no trânsito.

Este trabalho está estruturado em outras quatro seções: a próxima seção discorre sobre modelos categóricos, acidentes e fatores sociais; a seção 3 explana o método utilizado neste trabalho; a seção 4 trata da análise dos resultados e; a seção 5 traz as considerações finais do artigo.

\section{ACIDENTES E FATORES SOCIAIS}

Uma série de estudos recentes visa associar fatores sociais com a probabilidade de envolvimento em acidentes utilizando métodos econométricos. Factor et al. $(2007,2008)$ propuseram um modelo logit com fatores sociais para a ocorrência de acidentes em Israel, utilizando uma base de dados de acidentes cruzada à base do censo explorando a probabilidade de envolvimento em acidentes a partir de fatores socioeconômicos e demográficos, como gênero, idade, estado civil, religião, origem geográfica, renda, emprego e escolaridade. Os resultados mostram que mulheres, pessoas com mais de 60 anos e com escolaridade mais alta tem as menores chances de se envolver em acidentes fatais ou graves.

Borhan et al. (2018) encontraram resultados semelhantes para acidentes com motociclistas na Malásia, com relação à idade e escolaridade, no entanto, neste estudo a variável gênero não apresentou resultados significativos enquanto a variável renda apresentou. Os autores consideraram fatores sociais e de comportamento propenso ao risco. Concluíram que o comportamento propenso ao risco está relacionado ao uso da motocicleta e que existe uma diferença significativa entre a força da influência dos pares, pais e do cônjuge. Em Deli, na Índia, Goel et al. (2018) cruzaram dados de tráfego, com dados demográficos agregados do Censo (população, percentual da população empregada, taxa de alfabetização) e características estruturais da via (ex. número de paradas de ônibus, de rotatória) para avaliar a influência dos fatores usando uma abordagem bayesiana hierárquica com um modelo de regressão de Poisson-lognormal. Assim como Borham et al. (2018) e Factor et al. (2007, 2008), encontraram que o risco de fatalidade tem uma associação negativa com a escolaridade (taxa de alfabetização). Também tiveram relação negativa, densidade populacional e número de rotatórias, enquanto o porcentual da população empregada, número de pontos de ônibus, número de viadutos e quilômetros percorridos por veículos tiveram uma associação positiva.

Crundall et al. (2013) desenvolveram um estudo tipo survey para explorar a relação entre o risco do acidente e a quantidade de $\mathrm{km}$ rodados, idade, experiência, tipo de viagem, treinamento, características pessoais e comportamentais dos condutores. Os principais resultados indicam que a quantidade de quilômetros percorrida não foi significativa, o aumento da idade e da experiência em pilotar diminuem o risco de acidentes e que motos maiores tendem a uma maior probabilidade de envolvimento em acidentes mais graves. Usando redes bayesianas, De Oña et al. (2013) avaliaram acidentes em estradas rurais de Granada. Das variáveis analisadas, mês, hora, dia, tipo de acidente, causa, idade, gênero, largura do pavimento, sinalização e distância de visibilidade foram significativas para todos os casos analisados.

No Brasil, Coutinho et al. (2015) usaram modelos probit e logit para determinar fatores associados a acidentes de motociclistas em Fortaleza. Foram consideradas na análise as variáveis capacete, luz do dia, idade, final de semana, sexo, se o veículo era alugado e superfície da via, sendo que as três últimas listadas não foram significativas. Berger (2014) utilizou modelos lineares generalizados na investigação de fatores de risco e proteção associados à gravidade da 
ocorrência com motocicletas em Vitória. As variáveis analisadas foram idade, sexo, escolaridade, raça e local de residência, local de ocorrência, zona de ocorrência, horário, dia da semana, tipo de vítima, veículos, forma de socorro, ocorrência durante ou a caminho do trabalho, uso de álcool, uso de equipamentos de segurança, natureza da lesão, partes do corpo atingidas e evolução da emergência. Assim como na análise de Coutinho et al. (2015) os resultados demonstram a existência de relação do horário, dia da semana, além dessas duas variáveis, a parte do corpo atingida, ocorrência em zona rural ou urbana e município de ocorrência pertencer à zona metropolitana foram variáveis relacionadas com a chance de internação hospitalar e ocorrência de acidentes.

Liberatti et al. (2003) usaram análise multivariada para encontrar fatores associados ao não uso do capacete na hora do acidente em Londrina. Os fatores considerados no estudo foram sexo, idade, posição no veículo, presença de hálito etílico, dia da semana, horário e região do acidente. Foram mais fortemente significativos idade e presença de hálito etílico e não foram significativas sexo e posição da vítima. Silva (2015) usou a regressão de fatores macroeconômicos para determinar os municípios com maiores chances de altos coeficientes de mortalidade por acidentes de motocicleta. A pesquisa relatou que elevados fatores de crescimento populacional e aumentos na frota total de motocicletas, com baixa densidade populacional, baixo PIB per capita e mais de 20 motocicletas por mil habitantes foram as variáveis que demonstraram ter maior força em explicar maiores chances de morte de motociclistas.

Entretanto, nenhum dos estudos brasileiros encontrados na literatura busca relacionar diretamente fatores socioeconômicos, como escolaridade, renda e nível de educação no trânsito, à probabilidade de envolvimento em acidentes graves. 0 acesso a tais informações permite aos gestores públicos traçar um perfil dos indivíduos com maior probabilidade de envolvimento em acidentes graves e pautar políticas públicas específicas de combate à mortalidade por acidentes de transporte com motociclistas de maneira mais eficaz. É preciso ressaltar, no entanto, que a forma como o sistema de trânsito é organizada contribui para a acidentalidade dos usuários mais vulneráveis, dentre eles os motociclistas, e que, portanto, são necessárias também políticas pautadas para os demais usuários, notadamente para os motoristas de veículos maiores.

\section{METODOLOGIA}

Esta seção discute o método adotado neste estudo. A primeira subseção descreve o local onde a pesquisa foi realizada, seguida de subseções que discutem a amostra e a coleta de dados, o modelo adotado e suas variáveis.

\subsection{Local de pesquisa}

A área geográfica selecionada para este estudo foi o estado de Alagoas devido à alta frequência de acidentes com motocicleta. Alagoas possui 40\% da sua frota composta por motocicletas (DENATRAN, 2019), porém os usuários de motocicleta correspondem a 53\% dos óbitos por acidentes de transporte no estado (SESAU, 2019). Alagoas também está situado no Nordeste, região que concentra a segunda maior quantidade de indenizações por acidentes fatais (32\%) no Brasil, sendo a maior parcela (67\%) por motocicleta. Considerando as indenizações por invalidez, entre 2000 e 2011 as solicitações na Região Nordeste cresceram de 2.187 para 37.976 casos, representando um acréscimo de 1.636\% em apenas 11 anos (Seguradora Líder, 2018). 


\subsection{Amostra e coleta de dados}

Este estudo adotou um método quantitativo usando uma base de dados transversais de acidentados que buscaram obter o valor do seguro DPVAT. A base de dados foi criada com base nos questionários coletados pelo departamento de serviço social do DETRAN-AL. Quando o órgão é procurado para solicitação do seguro DPVAT, os solicitantes (o acidentado ou um parente) são convidados a preencherem um formulário social. As informações fornecidas relativas ao acidente (ex. uso de capacete) são confrontadas com as informações constantes no formulário de atendimento do SAMU e laudo médico, também anexados ao processo. 0 formulário utilizado para a coleta dos dados se mantém o mesmo desde 2013. Os dados a partir de 2016 estavam informatizados e foram obtidos via Lei de Acesso à Informação. Os dados de 2013 e 2014 foram compilados a partir do arquivo físico do DETRAN-AL nos meses de abril e maio de 2019 . 0 ano de 2015 foi excluído da amostra por ter sido o ano em que o DETRAN mudou de sede, causando interrupções nos atendimentos e na documentação. Foram obtidas 1220 amostras no total entre os anos de 2013 e 2019, excluindo-se 2015, sendo 355 em 2013, 203 em 2014, 219 em 2016, 214 em 2017, 200 em 2018, 29 em 2019 (até abril). Ao final, foram utilizados 689 questionários para análise, considerando-se apenas aqueles relativos aos acidentes com motocicletas.

\subsection{Variáveis}

O formulário utilizado pelo DETRAN-AL para dados dos solicitantes do DPVAT é dividido em 4 partes: i) dados socioeconômicos (escolaridade, situação ocupacional antes e após o acidente, renda, tipo de solicitação (DAMS - ressarcimento dos custos médicos, por deficiência permanente ou por morte); ii) dados pessoais (nome, sexo, data de nascimento, naturalidade, estado civil e endereço); iii) condição familiar antes e após o acidente, percentual de contribuição na renda familiar antes do acidente, recebimento de programa social); iv) dados do acidente (data, endereço, tipo de envolvimento da vítima - ex: condutor, tempo de habilitação, uso de equipamento de segurança e natureza - ex: colisão).

Tabela 1 - Estatística descritiva das variáveis do grupo de usuários e de condutores de motocicleta

\begin{tabular}{|c|c|c|c|c|c|}
\hline \multirow[b]{2}{*}{ Variável } & \multicolumn{2}{|c|}{ Usuários ( $\mathrm{N}=689)$} & \multicolumn{2}{|c|}{ Condutores $(\mathrm{N}=522)$} & \multirow[b]{2}{*}{ Descrição } \\
\hline & Prop. & Erro Padrão & Prop. & Erro Padrão & \\
\hline gravidade & 0,136 & 0,013 & 0,142 & 0,015 & 1 = se morte; 0 = se DAMS ou invalidez. \\
\hline $\operatorname{sexo}(M)$ & 0,868 & 0,013 & 0,941 & 0,010 & $1=$ se masculino, 0 = se feminino. \\
\hline \multicolumn{6}{|l|}{ idade } \\
\hline$\leq 29$ & 0,411 & 0,019 & 0,404 & 0,021 & $0=0$ a 29 anos \\
\hline $30-39$ & 0,326 & 0,018 & 0,350 & 0.021 & 1 = de 30 a 39 anos; \\
\hline $40-49$ & 0,155 & 0,014 & 0,142 & 0.015 & 2 = de 40 a 49 anos; \\
\hline$\geq 50$ & 0,107 & 0,012 & 0,107 & 0.013 & $3=$ maior ou igual a 50 anos; \\
\hline estado civil & 0,348 & 0,018 & 0,343 & 0,021 & $1=$ se solteiro, 0 = se outros. \\
\hline escolaridade & & & & & 1 = ensino médio completo, 0 = até ensino médio \\
\hline & 0,334 & 0,018 & 0,362 & 0,021 & incompleto. \\
\hline Renda & & & & & $0=$ menos de 1 S.M.; \\
\hline < 1 S.M. & 0,071 & 0,010 & 0,057 & 0,010 & $0=<1$ S.M. \\
\hline $1 \mathrm{~S}, \mathrm{M}$ & 0,234 & 0,016 & 0,237 & 0.019 & 1 = 1 S.M.; \\
\hline 1 a $2 S, M$ & 0,422 & 0,019 & 0,427 & 0.022 & 2 = mais de 1 a 2 S. M.; \\
\hline 2 a $3 \mathrm{~S}, \mathrm{M}$ & 0,180 & 0,015 & 0,073 & 0.017 & 3 = mais de 2 a 3 S.M.; \\
\hline 3 A 5 S,M & 0,068 & 0,010 & 0,029 & 0.011 & $4=$ mais de 3 a 5 S.M. \\
\hline+ de $5 \mathrm{~S}, \mathrm{M}$ & 0,025 & 0,006 & 0,029 & 0.007 & $5=$ mais de 5 S.M. \\
\hline tipo de motocicleta & 0,853 & 0,013 & 0,868 & 0,015 & $1=>50 \mathrm{cc}, 0=<50 \mathrm{cc}$ \\
\hline capacete & 0,898 & 0,011 & 0,925 & 0,011 & $1=$ sem capacete, $0=$ com capacete \\
\hline habilitação & - & - & 0,274 & 0,019 & $1=$ sem habilitação, $0=$ habilitado \\
\hline final de semana & 0,383 & 0,018 & 0,375 & 0,021 & $1=$ final de semana, $0=$ durante a semana \\
\hline
\end{tabular}


As variáveis socioeconômicas usadas na modelagem foram: sexo, idade, estado civil, escolaridade e renda. Também foram consideradas variáveis relacionadas ao acidente, como: tipo de motocicleta, uso do capacete, final de semana e habilitação (se condutores). Para o processo de calibração do modelo, as variáveis foram codificadas em variáveis categóricas, cuja descrição e estatísticas envolvidas no estudo para os grupos de usuários de motocicletas e de condutores de motocicleta constam na Tabela 1.

\subsection{Modelo}

A variável dependente tem resposta binária, portanto foi utilizado um modelo logit, brevemente explanado abaixo, para mais detalhes consultar Wooldridge (2010). Partiu-se da seguinte equação:

$$
P(y=1 \mid \mathbf{x})=\mathrm{G}(\mathbf{x} \boldsymbol{\beta})=\frac{\mathrm{e}^{\mathrm{x} \boldsymbol{\beta}}}{1+\mathrm{e}^{\mathrm{x} \boldsymbol{\beta}}}
$$

onde $\mathrm{P}(\mathrm{y}=1 \mid \mathbf{x})$ corresponde à probabilidade de morte condicional ao vetor de variáveis explicativas (x), e representa o exponencial, enquanto G representa uma função de distribuição cumulativa logística, função que permite calcular a probabilidade predita. Uma das vantagens desse tipo de modelo comparado aos Mínimos Quadrados Ordinários (conhecido como modelo de probabilidade linear) é que ele garante que as probabilidades pertençam ao intervalo entre $0 \mathrm{e}$ 1. Um modelo alternativo, probit, que considera uma distribuição normal padrão geraram resultados bastante semelhantes e podem ser obtidos através de contato com os autores.

Os parâmetros da Equação (1) são obtidos através do estimador de Máxima Verossimilhança (MV), para tanto, o estimador maximiza a função log verossimilhança (Equação 2), considerando o somatório de todas as $n$ observações e a distribuição logística:

$$
l=\sum_{i=1}^{n}\left\{y_{i} \log \left[G\left(\mathbf{x}_{i} \boldsymbol{\beta}\right)\right]+\left(1-y_{i}\right) \log \left[1-G\left(\mathbf{x}_{i} \beta\right)\right]\right\}
$$

As probabilidades foram preditas de acordo como a equação (1) e estimativas via MV, sendo que essas probabilidades dependem do nível das variáveis explicativas. Considerando que as variáveis utilizadas no artigo são categóricas, a probabilidade predita é mensurada através da presença da característica em questão (por exemplo, ser do sexo feminino) e para as demais variáveis ( $\mathbf{x}$ ) assumindo os valores referentes a cada indivíduo, e posteriormente, obtendo a média, assim, obtêm-se uma probabilidade predita média.

Os efeitos marginais foram obtidos simplesmente subtraindo a probabilidade predita média da característica em questão comparativamente à predição para categoria base (por exemplo, sexo feminino e masculino). Por fim, as razões de chances foram obtidas calculando o exponencial dos coeficientes estimados do logit.

0 ajuste do modelo foi analisado através de: i) um teste qui-quadrado comparando um modelo irrestrito (todas as variáveis explicativas) com o modelo restrito (modelo nulo, sem variáveis); ii) o teste de Hosmer-Lemeshow, a não rejeição da hipótese nula de que frequências observadas e esperadas sejam iguais indica um bom ajuste; iii) Também foi reportado o Pseudo R2. Porém, cabe destacar que este último indicador deve ser interpretado com cautela, já que não existe um patamar mínimo necessário para um bom ajuste, como observado em Wooldridge (2010), mais do que o ajuste, a preocupação deve focar na obtenção de efeitos ceteris paribus. Assim, deve-se destacar algumas limitações como possíveis variáveis omitidas ou algum viés amostral que podem ter efeito sobre os resultados desta análise. Conquanto, as contribuições da análise no sentido de compreender quais fatores são significativos para a gravidade de acidentes são de grande valia para o embasamento políticas públicas de transporte e prevenção de acidentes. 
Ademais, para obter um modelo mais parcimonioso mantendo apenas variáveis com algum poder de explicação, na seleção de variáveis foi utilizado o método stepwise com eliminação backward. Considerando um modelo completo com todos os indicadores, a cada etapa, uma variável é considerada para subtração (a que possuir o maior valor-p), o modelo final é obtido quando não há nenhuma variável a ser excluída dado o nível de significância escolhido, no caso deste trabalho $10 \%$.

\section{ANÁLISE DOS RESULTADOS}

Neste estudo, os dados foram analisados em dois modelos diferentes, como mencionado anteriormente. No primeiro modelo, foram utilizados os dados do total de motociclistas presentes na amostra ( $\mathrm{N}=689)$. Este modelo é retratado na seção 4.1 e o grupo é denominado "Usuários", pois trata-se das pessoas que se acidentaram estando numa motocicleta seja como passageiro ou como piloto. No segundo modelo, foram selecionados apenas os dados referentes a condutores de motocicletas $(\mathrm{N}=522)$, para capturar o efeito da habilitação sobre o aumento da gravidade dos acidentes. Este grupo é avaliado na seção 4.2 denominada "Grupo de condutores".

\subsection{Grupo de Usuários}

A Figura 1 apresenta as probabilidades preditas baseadas no modelo logit após escolha das variáveis via stepwise. Cabe destacar que para as variáveis renda e idade, também foram testadas se as diferentes categorias eram conjuntamente estatisticamente iguais a zero, em ambos os casos a hipótese nula não pode ser rejeitada. Já a tabela 2 apresenta os coeficientes do modelo logit, o ajuste do modelo e os efeitos marginais (diferenças entre as probabilidades preditas). Primeiramente, cabe destacar que o valor do McFadden Pseudo R-Quadrado foi de 0,1284 e o valor do Teste Chi-Quadrado foi de 62,77, ou seja, o modelo é conjuntamente estatisticamente significativo. Além disso, o teste Hosmer-Lemeshow indicou que não podemos rejeitar a hipótese nula de que frequências observadas e esperadas sejam iguais, indicando um bom ajuste. Em relação à significância estatística, todas as outras cinco variáveis que permaneceram no modelo possuem efeitos marginais significativos a um nível de $5 \%$.

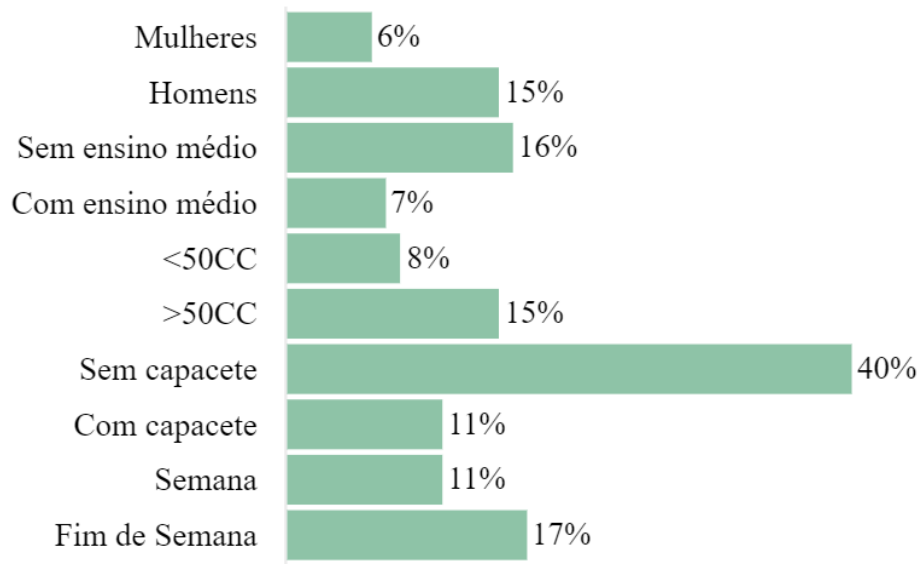

Figura 1. Probabilidade de envolvimento em acidentes com morte para variáveis significativas

Observa-se que, considerando o grupo total de usuários, apesar de diferenças em educação, gênero, cilindrada e dia de ocorrência, as chances de envolvimento são significativamente maiores para usuários sem capacete, cuja probabilidade estimada de envolvimento em acidente 
com morte é de 40\%. Ou seja, dado que as demais variáveis estejam fixadas na média, a probabilidade de morte é 3 vezes maior sem o uso do equipamento de segurança.

De acordo com este modelo, homens têm mais do que o dobro de probabilidade de se envolverem em um acidente fatal, assim como os usuários de motos de cilindradas maiores (> 50cc). Considerando o efeito da educação, a probabilidade de envolvimento em um acidente fatal é menor à medida em que o grau de escolaridade se eleva, passando de $16 \%$ para indivíduos sem ensino médio, para 7\% entre os usuários que terminaram o segundo grau. Existe ainda, uma probabilidade de envolvimento em acidente fatal 4 pontos percentuais maior aos finais de semana.

Tabela 2 - Regressão logística em acidentes graves e fatais em variáveis sociais e de controle, grupo usuários de motocicleta

\begin{tabular}{|c|c|c|c|c|}
\hline y & Coef. & Erro Padrão & Efeitos Marginais & Erro Padrão \\
\hline Sexo (M) & $1,083^{* *}$ & 0,532 & $0,086^{* * *}$ & 0,030 \\
\hline Escolaridade & $-0,990^{\star * *}$ & 0,321 & $-0,091^{* * *}$ & 0,025 \\
\hline Moto + 50cc & $0,805^{*}$ & 0,455 & $-0,070^{* *}$ & 0,032 \\
\hline Capacete & $-1,861^{\star * *}$ & 0,371 & $-0,291^{* * *}$ & 0,069 \\
\hline Final de Semana & $0,513^{* *}$ & 0,244 & $0,055^{\star *}$ & 0,027 \\
\hline Constante & $-2,229^{\star * *}$ & 0,579 & - & - \\
\hline & $\chi^{2}=62.77$ & Pseudo $R^{2}$ & $\chi^{2}=10,30$ & \\
\hline Ajuste & $(p=0,000)$ & 0,1284 & $(p=0,172)$ & \\
\hline
\end{tabular}

\subsection{Grupo de Condutores}

A Figura 2 e Tabela 3 mostram cálculos similares à Figura 1 e Tabela 2, sendo que considerando o grupo de condutores. Este grupo foi separado de modo a permitir a observação do efeito da educação formal no trânsito (ser habilitado) na probabilidade de envolvimento em acidentes fatais.

No ajuste final do modelo logit, cinco variáveis permaneceram após a aplicação do stepwise para seleção de variáveis. Assim como no caso anterior, o modelo é conjuntamente estatisticamente significativo e o teste Hosmer-Lemeshow indicou um bom ajuste do modelo. Novamente, as variáveis renda e idade apresentaram categorias que conjuntamente possuem efeitos iguais a zero.

Nota-se que para este grupo, os seguintes efeitos marginais são significativos a 5\%: escolaridade, cilindrada, uso de capacete e habilitação. 0 efeito marginal do final de semana foi significativo a $10 \%$. Diferentemente do modelo anterior, a variável sexo não se mostrou significativa. Destaca-se a ausência de capacete com uma probabilidade de $41 \%$ de morte contra 12\% para os que o utilizavam. Estima-se que os condutores de motocicletas, em comparação com os condutores de ciclomotores, têm uma probabilidade de morte 2,4 vezes maior, isto pode ser um reflexo indireto das diferenças de velocidades máximas alcançadas pelos veículos.

Mais uma vez, o grau de educação se mostra representativo, sendo a probabilidade de envolvimento em acidentes graves para indivíduos que não chegaram a terminar o ensino médio o dobro da probabilidade dos que o concluíram. Relativamente ao dia da semana, condutores que se acidentam aos finais de semana, têm uma probabilidade de morte de 18\%, enquanto pessoas 
que se acidentam durante os dias úteis têm probabilidade de $12 \%$. Constatou-se que não-habilitados possuem uma probabilidade 13,3 pontos percentuais mais elevada de morte.

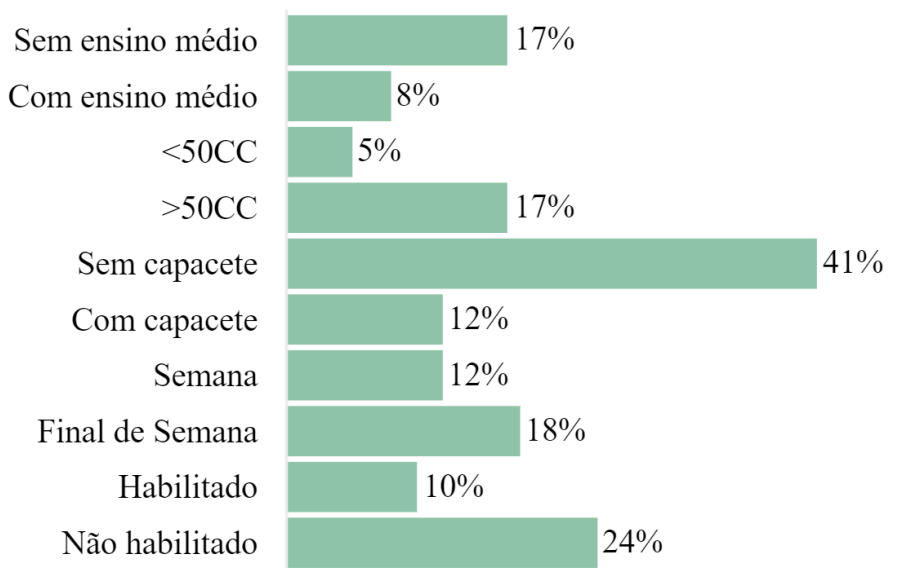

Figura 2. Probabilidade de envolvimento de condutores em acidentes fatais para variáveis significativas

Tabela 3 - Regressão logística em acidentes graves e fatais em variáveis sociais e de controle, grupo condutores

\begin{tabular}{|c|c|c|c|c|}
\hline$y$ & Coef. & Erro Padrão & Efeitos Marginais & Er. Padrão \\
\hline Escolaridade & $-1,076^{\star * *}$ & 0,373 & $-0,097^{\star * *}$ & 0,029 \\
\hline Moto + 50cc & $1,648^{* * *}$ & 0,587 & $0,122^{\star * *}$ & 0,028 \\
\hline Capacete & $-1,852^{\star * *}$ & 0,476 & $-0,287^{* * *}$ & 0,092 \\
\hline Final de Semana & $0,571^{* *}$ & 0,281 & $0,060^{*}$ & 0,031 \\
\hline Habilitação & $1,117^{\star \star \star}$ & 0,318 & $0,133^{\star \star *}$ & 0,044 \\
\hline Constante & $-2,169^{\star * *}$ & 0,539 & - & - \\
\hline Ajuste & $\begin{array}{l}\chi^{2}=71,56 \\
(p=0,000)\end{array}$ & $\begin{array}{l}\text { Pseudo } \\
R^{2}=0,1679\end{array}$ & $\begin{array}{l}\chi^{2}=3,05 \\
(p=0,881)\end{array}$ & \\
\hline
\end{tabular}

\section{DISCUSSÃO}

As características significativas podem ser divididas em dois grupos: a) atributos de condução e b) atributos sociais.

\subsection{Atributos de Condução}

São os que estão ligados ao acidente em si e ao ato de conduzir. Como esperado, e também, como encontrado por Coutinho et al. (2015), Berger (2014) e Liberatti (2003), a probabilidade de óbito é mais elevada para usuários que não utilizavam capacete. Usuários de motocicletas maiores, acima de 50 cc, também são mais propensos a acidentes graves de acordo com a modelagem executada mas, na literatura, estudos divergem sobre os acidentes com motocicletas serem mais fatais do que com motonetas (Haworth et al. 2009).

No grupo de condutores, o fato da pessoa não ser habilitada também foi um fator significativo para o aumento da probabilidade de morte, assim como encontrado por Sexton et al. (2004) e Crundall et al. (2013). 0 fato de ocorrências nos finais de semana serem mais fatais corrobora com conclusão semelhante levantada por Coutinho et al. (2015), Berger (2014) e Liberatti (2003). Ou seja, considerando as características de condução, os fatores que influenciam na 
gravidade do acidente para os motociclistas em Alagoas são semelhantes aos fatores encontrados em estudos nacionais e internacionais.

\subsection{Atributos Sociais}

Estão relacionados às características pessoais do indivíduo. No grupo dos usuários gerais os acidentes mais graves estão relacionados com as variáveis escolaridade e sexo. Indivíduos com pelo menos o ensino médio completo têm menos chance do que indivíduos que não chegaram a concluir o ensino médio de se envolver em acidentes graves, como também encontrado em Borhan et al. (2018) e Goel et al. (2018).

Também foi avaliado que mulheres têm uma probabilidade menor do que homens de se envolverem em acidentes fatais, corroborando com Factor et al. (2008) e De Oña (2011). Nenhum dos estudos brasileiros revisados encontrou significância para sexo. É importante destacar que esta variável foi significativa apenas considerando o grupo total de usuários, mas não para o grupo de condutores. É possível que o pequeno número de observações de mulheres condutoras de motocicleta envolvidas em acidentes fatais na base utilizada para a análise tenha contribuído para este resultado. É possível também, que esta seja a razão pela qual a variável não foi significativa nos demais estudos nacionais

Com relação à idade, a variável não se mostrou significativa neste estudo em concordância com o apontado por Liberatti (2003) e Berger (2014). Nos estudos onde a variável se mostrou significativa, há divergências neste sentido com alguns estudos apontando que os mais jovens estão mais propensos a acidentes graves (De Oña, 2011; Coutinho et al. 2015; Borhan et al. 2018) e, outros, cujos resultados mostram que idades mais avançadas tendem a uma maior fatalidade (Chang e Yeh, 2006). A renda, embora estivesse nas variáveis avaliadas, não foi significativa de acordo com o modelo, diferindo dos estudos de Factor et al. (2008) e Borhan et al. (2018) para os quais a variável foi significativa.

\section{CONCLUSÃO}

Os resultados desta pesquisa indicam diferenças significativas na probabilidade de envolvimento de motociclistas em acidentes graves e fatais, de acordo com fatores sociais e fatores relacionados ao acidente. Por exemplo, a probabilidade de envolvimento encontrada é mais alta para homens do que para mulheres, para pessoas com escolaridade abaixo do ensino médio completo do que para pessoas que concluíram o segundo grau, para motocicletas acima de 50cc do que para 'cinquentinhas', para o usuário sem capacete do que para o que utiliza o equipamento e para acidentes nos finais de semana do que para acidentes durante a semana, para o condutor não habilitado do que para o com habilitação. A maior frequência de acidentes nos finais de semana pode estar relacionada ao uso de álcool associado à direção, porém não podemos afirmar se esta relação existe na amostra, pois esta variável não pôde ser controlada no estudo.

Desta forma, os resultados além de expor as diferenças entre os grupos com características diferentes, reforçam que os acidentes são distribuídos de maneira desigual entre os grupos sociais e podem ser socialmente construídos (no caso das diferenças entre homens e mulheres) ou relacionados ao grau de instrução (escolaridade/habilitação).

Este trabalho mostra que grupos socialmente desfavorecidos são mais vulneráveis a acidentes. Tal resultado pode ser percebido pela associação entre a condição socioeconômica do usuário de motocicleta com o envolvimento em acidentes mais graves em ambos os grupos 
avaliados - quanto maior o grau de educação, menor a probabilidade de envolvimento em acidente fatal. Dentre a literatura nacional, não foram encontrados estudos econométricos com microdados que abordassem especificamente a importância de estudar os fatores sociais; no entanto, resultados semelhantes foram encontrados em literatura estrangeira (Factor et al. 2008; Haque et al. 2010; Borhan et al. 2018). Assim, a principal contribuição deste artigo é trazer ao cenário brasileiro a abordagem social do fenômeno da segurança viária.

No entanto, vale salientar que a análise limita-se ao grupo de solicitantes do DPVAT em Alagoas. Seriam desejáveis análises mais abrangentes para o total de usuários das vias para prever as probabilidades reais de envolvimento em acidentes e não apenas a probabilidade de envolvimento em acidentes graves, dado que se acidentou. Além disso, deve-se considerar limitações relativas a possíveis variáveis omitidas ou algum viés amostral que possam ter exercido efeito sobre os resultados desta análise.

0 envolvimento de um membro provedor em um acidente grave pode levar a família a perder a sua fonte de renda principal. Seria interessante estudos que buscassem medir o impacto do acidente na renda dessas famílias e o nível de vulnerabilidade financeira familiar decorrente do acidente bem como pesquisas qualitativas em profundidade ou grupos focais que auxiliem na compreensão dos mecanismos que levariam determinados grupos a terem uma maior probabilidade de envolvimento em acidentes graves.

Uma vez que as características particulares dos grupos de indivíduos fossem exploradas seria possível, por exemplo, desenvolver programas educacionais específicos para cada um deles. Estes programas devem ser desenhados com o auxílio de integrantes do grupo e especificamente para cada um deles, de forma a suprir as necessidades cognitivas particulares a eles. Acredita-se que programas focados nos grupos de maior risco teriam uma maior efetividade na conscientização da população e consequente redução dos acidentes do que programas destinados à população em geral.

\section{AGRADECIMENTOS}

As autoras e o autor agradecem ao DETRAN-AL por ter gentilmente cedido a sua base de dados para esta pesquisa.

\section{REFERÊNCIAS}

Amoros, E.; J. Martin e B. Laumon (2006) Under-reporting of road crash casualties in France. Accident analysis and prevention 38, 627-635. DOI: 10.1016/j.aap.2005.11.006

Berger, P. (2014) Estudo dos Acidentes com Motocicletas e da Gravidade das Lesões Decorrentes. Dissertação (mestrado). Engenharia Civil, Universidade Federal do Espírito Santo. Vitória. Disponível em: < https://repositorio.ufes.br/bitstream/10/3980/1/tese_8491_Disserta\%C3\%A7\%C3\%A3o\%20-\%20Pedro\%20Pelacani\%20Berger\%20Vers\%C3\%A3o\%20Impress\%C3\%A3o.pdf > (acesso em 21/06/2021).

Borhan, M. N. et al. (2018) The relationship between the demographic, personal, and social factors of Malaysian motorcyclists and risk-taking behavior at signalized intersections. Accident Analysis \& Prevention, v. 121, p. 94-100. DOI: 10.1016/j.aap.2018.09.004

Chang, H. e T. Yeh (2006) Risk factors to driver fatalities in single-vehicle crashes: comparisons between non-motorcycle drivers. Journal of Transportation Engineering. ASCE 132 (3), 227-236. DOI: 0.1061/(ASCE)0733-947X(2006)132:3(227)

Coutinho, C. H. L.; F. J. C. Cunto e S. M. P. Ferreira (2015) Análise da severidade dos acidentes com motocicletas utilizando modelos probit e logit ordenados. Transportes, 23(4), 60-66. DOI: 10.14295/transportes.v23i4.926

Crundall, D.; E. Van Loon; A. W. Stedmon e E. Crundall (2013) Motorcycling experience and hazard perception. Accident Analysis \& Prevention, 50, 456-464. DOI: 10.1016/j.aap.2012.05.021

De Oña, J.; G. Lopez; R. Mujalli e F.J. Calvo(2013) Analysis of traffic accidents on rural highways using latent class clustering and Bayesian networks. Accident Analysis \& Prevention 51, 1-10. DOI: 10.1016/j.aap.2012.10.016

DENATRAN - DEPARTAMENTO NACIONAL DE TRÂNSITO (2019) Frota de veículos, por tipo e com placa, segundo as Grandes Regiões e Unidades da Federação - Fev/2019. Disponível em: < https://www.gov.br/infraestrutura/pt-br/assuntos/transito/conteudo-denatran/frota-de-veiculos-2019>. Acesso em: 21/06/2021. 
Factor, R.; D. Mahalel e G. Yair (2007) The social accident: A theoretical model and a research agenda for studying the influence of social and cultural characteristics on motor vehicle accidents. Accident Analysis \& Prevention, 39(5), 914-921. DOI: 10.1016/j.aap.2006.12.015

Factor, R., D. Mahalel e G. Yair (2008) Inter-group differences in road-traffic crash involvement. Accident Analysis \& Prevention, 40(6), 2000-2007. DOI: 10.1016/j.aap.2008.08.022

Goel, R.; P. Jain e G. Tiwari (2018) Correlates of fatality risk of vulnerable road users in Delhi. Accident Analysis \& Prevention, v. 111, p. 86-93. DOI: 10.1016/j.aap.2017.11.023

Haque, M.M.; C.C. Hoong e C.L. Beng (2010) Effects of impulsive sensation seeking, aggression and risk-taking behavior on the vulnerability of motorcyclists. Asian Transport Studies 1 (2), 165-180. DOI: 10.11175/eastsats.1.165

Haworth, N.; K. Greig e A. Nielson (2009) A comparison of risk taking in moped and motorcycle crashes. Transportation Research Record. 2140, 182-187. DOI: 10.3141/2140-20

Liberatti, C. L. B.; S. M. D. Andrade; D. A. Soares e T. Matsuo (2003) Uso de capacete por vítimas de acidentes de motocicleta em Londrina, sul do Brasil. Revista Panamericana de Salud Pública, 13 (1), 33-38. Disponível em: <https://www.scielosp.org/pdf/rpsp/2003.v13n1/33-38/pt>. Acesso em: 17/06/2021.

MS - MINSTÉRIO DA SAÚDE, DEPARTAMENTO DE INFORMÁTICA DO SUS - DATASUS (2019) Informações de Saúde, Epidemiológicas e Morbidade: banco de dados. Óbitos por causas externas - Brasil. 2019. Disponível em: <http://tabnet.datasus.gov.br/cgi/tabcgi.exe?sim/cnv/ext10uf.def>. Acesso em: 17/06/2021.

OMS - ORGANIZAÇÃO MUNDIAL DA SAÚDE (2015) Global status report on road safety. OMS, Itália. Disponível em: < https://www.afro.who.int/sites/default/files/2017-06/9789241565066_eng.pdf>. Acesso em: 17/06/2021.

SESAU - SECRETARIA DE ESTADO DE SAÚDE DE ALAGOAS (2019) Mortalidade por acidentes de transporte terrestres. Boletim Informativo SUVISA Ano 3, n. 3, Maio 2019.

SEGURADORA LÍDER (2018) Boletim estatístico, agosto 2018. Disponível em: <https://www.seguradoralider.com.br/Documents/boletim-estatistico/BOLETIM-VOL08-AGOSTO-2018.pdf >. Acesso em: 17/06/2021.

Sexton, B. F.; C. J. Baughan; M. A. Elliott e G. Maycock (2004) The accident risk of motorcyclists. TRL Report TRL607, University of Strathclyde. Disponível em: <https://strathprints.strath.ac.uk/20274/1/strathprints020274.pdf> Acesso em: $17 / 06 / 2021$.

Silva, P. H. N. V. et al. (2015) Deaths due to motorcycle accidents and their association with variables related to social reproduction in a northeastern Brazilian state. Salud Colectiva, v. 11, n. 3, p. 401-410. DOI: 10.18294/sc.2015.724

Vlahogianni, E. I., Yannis, G., \& Golias, J. C. (2012) Overview of critical risk factors in Power-Two-Wheeler safety. Accident Analysis \& Prevention, 49, 12-22. DOI: 10.1016/j.aap.2012.04.009

Weinsefilz, J. (2013) Mapa da violência 2013: acidentes de trânsito e motocicletas. Rio de Janeiro, 2013. Disponível em: < http://flacso.org.br/files/2020/03/mapa2013_transito.pdf > Acesso em: 17/06/2021. 